\title{
Corrigendum
}

\section{Corrigendum to "Deep Learning in the Detection and Diagnosis of COVID-19 Using Radiology Modalities: A Systematic Review”}

\author{
Mustafa Ghaderzadeh (iD) ${ }^{1}$ and Farkhondeh Asadi (iD ${ }^{2}$ \\ ${ }^{1}$ Student Research Committee, Department and Faculty of Health Information Technology and Management, \\ School of Allied Medical Sciences, Shahid Beheshti University of Medical Sciences, Tehran, Iran \\ ${ }^{2}$ Department of Health Information Technology and Management, School of Allied Medical Sciences, \\ Shahid Beheshti University of Medical Sciences, Tehran, Iran \\ Correspondence should be addressed to Farkhondeh Asadi; asadifar@sbmu.ac.ir
}

Received 29 September 2021; Accepted 29 September 2021; Published 25 October 2021

Copyright (c) 2021 Mustafa Ghaderzadeh and Farkhondeh Asadi. This is an open access article distributed under the Creative Commons Attribution License, which permits unrestricted use, distribution, and reproduction in any medium, provided the original work is properly cited.

In the article titled "Deep Learning in the Detection and Diagnosis of COVID-19 Using Radiology Modalities: A Systematic Review" [1], the Acknowledgements section should be corrected as follows:

"This study is based on project no. 1399/61288 at the Student Research Committee, Shahid Beheshti University of Medical Sciences, Tehran, Iran. The authors appreciate the Student Research Committee and Research \& Technology Chancellor at Shahid Beheshti University of Medical Sciences for their financial support of this study."

\section{References}

[1] M. Ghaderzadeh and F. Asadi, "Deep learning in the detection and diagnosis of COVID-19 using radiology modalities: a systematic review," Journal of Healthcare Engineering, vol. 2021, Article ID 6677314, 10 pages, 2021. 